\title{
Disinfection of Microbes by Magnetized DC Plasma
}

\author{
Ahmed Rida Galaly ${ }^{1,2}$, Hamdi Hussien Zahran ${ }^{3}$ \\ ${ }^{1}$ Physics Department, Faculty of Science, Beni-Suef University, Beni-Suef, Egypt \\ ${ }^{2}$ Engineering Science Department, Faculty of Community, Umm Al-Qura University, \\ Makkah, KSA \\ ${ }^{3}$ Department of Botany, Faculty of Science, Beni-Suef University, Beni-Suef, Egypt \\ Email: ahmed_galaly@yahoo.com
}

Received 28 April 2014; revised 26 May 2014; accepted 15 June 2014

Copyright (C) 2014 by authors and Scientific Research Publishing Inc. This work is licensed under the Creative Commons Attribution International License (CC BY). http://creativecommons.org/licenses/by/4.0/

\section{(c) (i) Open Access}

\section{Abstract}

A different combined effects for helium gas discharge such as: magnetic field strength, breakdown voltage, applied power, applied pressure, cathode fall thickness, edge effect, distribution of the electron temperature and density, and finally exposure time for Staphylococcus aureus substrate over slides at the cathode edge, are discussed under the influence of cold, nonthermal plasmas, ultra low pressure, and presence of the magnetic field for disinfection of bacteria for short exposure times, compatible to International Commission on Non-Ionizing Radiation Protection, Health Phys (ICNIRP) for healing applications. Furthermore, analyses of the experimental data of initial and final densities of cells alive, using survival curves, showed an impressive inhibitory effect of plasma discharge to the remaining survival of bacterial ratio under the influence of the magnetic field.

\section{Keywords}

DC Glow, Magnetic Field, Cathode Fall, Edge Effect, Non-Ionizing Radiation Protection, Health Phys ICNIRP

\section{Introduction}

Healing application of plasma in the human body considers a challenge for both medicine and plasma physics. At present, concepts of appropriate plasma sources that collect the technical requirements of medical instrumentation are still undeveloped. To reach selected effects and to avoid potential risks, it is necessary to know how to control the composition and densities of reactive plasma components by external operation parameters. Therefore, a deep fact on plasma physics and chemistry must be contributed by physical research. As these plasmas 
are huge challenge for plasma diagnostics, it is necessary to supply the required knowledge on plasma sources for healing applications [1]. The application of a magnetic field on the discharge region results in enhancement of some attractive features of specific plasma sources [2]. Moreover there are many articles focusing on theoretical and experimental study of factors of the sterilization of medical articles in low pressure glow discharge plasma [3], furthermore sterilization of medical products in low-pressure glow discharges and showing that roles of the various plasma agents in the inactivation of bacteria become the new fashion for applications of plasma [4] [5]. Also there are other methods to determine inactivation factors of spore-forming bacteria by plasma discharge such as: using low-pressure microwave plasmas in gas mixture, using Low-Pressure Radio-Frequency Oxygen Plasma and by using air plasmas at atmospheric pressure [6] [7].

Of late, it has been an important method in many non-equilibrium glow discharge plasma used in inactivation processes of microorganisms. Many studies have been carried out on plasma in a magnetic field, but most of them were about the effect of Extremely Low-Frequency (ELF) magnetic fields on the bacterial cell [8], that is, in which the magnetic field is not strong enough to make an ion do a cyclotron motion in the sheath before it strikes the target.

The present study aims to demonstrate the performance of magnetized direct current (DC) plasma for helium gas discharge in the disinfection process of cells alive. The inactivation processes for Staphylococcus aureus in the cathode fall (CF) region in magnetized DC helium plasma is investigated under the influence of some parameters such as: different magnetic field strengths from strong permanent magnet, CF thickness, breakdown voltages, applied electric powers, applied gas pressures, and different exposure times for Staphylococcus aureus substrate over slides at the cathode edge, under the control of cold, non-thermal plasmas, ultra low pressure, and presence of the magnetic field for disinfection of bacteria for short exposure times and for complete sterilization, compatible to International Commission on Non-Ionizing Radiation Protection, Health Phys (ICNIRP) for healing applications.

\section{Experimental Setup}

\subsection{Electrical Circuit}

Figure 1(a) shows the magnetized DC plasma electrical circuit and the plasma cell. The DC plasma structure consists of a stainless steel chamber that has two glass ports, connected to vacuum system. The pump speed was about $10^{5}$ lit./sec. and the vacuum of a about $10^{-5}$ torr at room temperature. An oil trap was used to eliminate the impurities from reaching the discharge system. During all measurements, a continuous gas flow through the discharge vessel was maintained to sweep out the impurities. The gas was fed to the discharge tube using a needle valve. The pressure of the system was measured using two types of gauges, a dial gauge (Edward's type CG3) which covers the range from 20 to $0.1 \mathrm{mbar}$ and a thermocouple gauge type 77MT-2, covers the range from 0.5 down to $5 \times 10^{-5}$ torr.

Figure 1(b) shows The geometry and the dimension of two hollow permanent circular magnets are placed bounded the cathode surface to produce the magnetic field, Each of them has a maximum magnetic field strength of 200 Gauss and with radial diameter $80 \mathrm{~mm}$ and hollow at the center of the magnet for the cathode position with the same diameter $50 \mathrm{~mm}$, where the radial and axial distribution for the magnetic field strengths as measured by MG-4D Hall probe, which is a fully moveable hand-held Hall effect gauss meter.

\subsection{Microbial Material Reaction with Plasma and Preparing}

Biological material can be exposed to plasma by direct exposure, where the treated slides position is at the cathode edge (containing Staphylococcus aureus bacteria) are in direct contact with the magnetized DC plasma. All plasma generates agents, including charged particles, and come in contact with the sample (slides containing bacteria). As the amount of heat transmitted to the sample is increased, the charged particles play a role as they sputter the sample, and many of the short-lived neutral reactive species also reach the sample.

To show a clear illustration effect of the plasma discharge on inactivation process of Staphylococcus aureus bacterium, and to express it in an observable way, a hollow permanent magnet is placed surrounded the cathode to increase the inactivation efficiency.

\subsubsection{Isolation and Cultivation of Bacteria}

The bacteria Staphylococcus aureus will be isolated from clinical specimens of human skin, mucosa and sputum, 
collected from patients. The cultured bacteria on agar plates will be identified according to the appearance of colonies, growth conditions and metabolic enzymatic activities.

\subsubsection{Preparing Staphylococcus aureus Microbes Slides \\ a. Mannitol Salt Agar}

Mannitol Salt Agar is a special bacterial growth medium that is selective for halophiles and can differentiate pathogenic and nonpathogenic Mannitol Salt Agar is used for the isolation of staphylococci. Chapman formulated Mannitol Salt Agar to isolate staphylococci by inhibiting growth of most other bacteria with a high salt concentration. Chapman added 7.5\% Sodium Chloride to Phenol Red Mannitol Agar and noted pathogenic strains of staphylococci (coagulase-positive staphylococci) grew luxuriantly and produced yellow colonies with yellow zones [9]. Nonpathogenic staphylococci produced small red colonies with no color change to the surrounding medium. Mannitol Salt Agar is highly selective, and specimens from heavily contaminated sources may be streaked onto this medium without danger of overgrowth. Mannitol Salt Agar is recommended for isolating pathogenic staphylococci from clinical specimens, cosmetics, and microbial limit tests.

\section{b. Principles of the microbiological sample Procedure}

Principles of the Procedure depend on Enzymatic Digest of Casein, Enzymatic Digest of Animal Tissue, and Beef Extract provides the nitrogen, vitamins, and carbon in Mannitol Salt Agar. D-Mannitol is the carbohydrate source. In high concentrations, Sodium Chloride inhibits most bacteria other than staphylococci. Phenol Red is the $\mathrm{pH}$ indicator. Agar is the solidifying agent. Bacteria that grow in the presence of a high salt concentration and ferment mannitol produce acid products, turning the Phenol Red $\mathrm{pH}$ indicator from red to yellow. Typical pathogenic staphylococci ferment mannitol and form yellow colonies with yellow zones. Typical non-pathogenic staphylococci do not ferment mannitol and form red colonies [10].

\begin{tabular}{cc}
\hline Formula & (g/l) \\
\hline Enzymatic Digest of Casein & 5 \\
Enzymatic Digest of Animal Tissue & 5 \\
Beef Extract & 1 \\
D-Mannitol & 10 \\
Sodium Chloride & 75 \\
Phenol Red & 0.025 \\
Agar & 15 \\
Final pH: & $7.4 \pm 0.2$ at $25^{\circ} \mathrm{C}$ \\
\hline
\end{tabular}

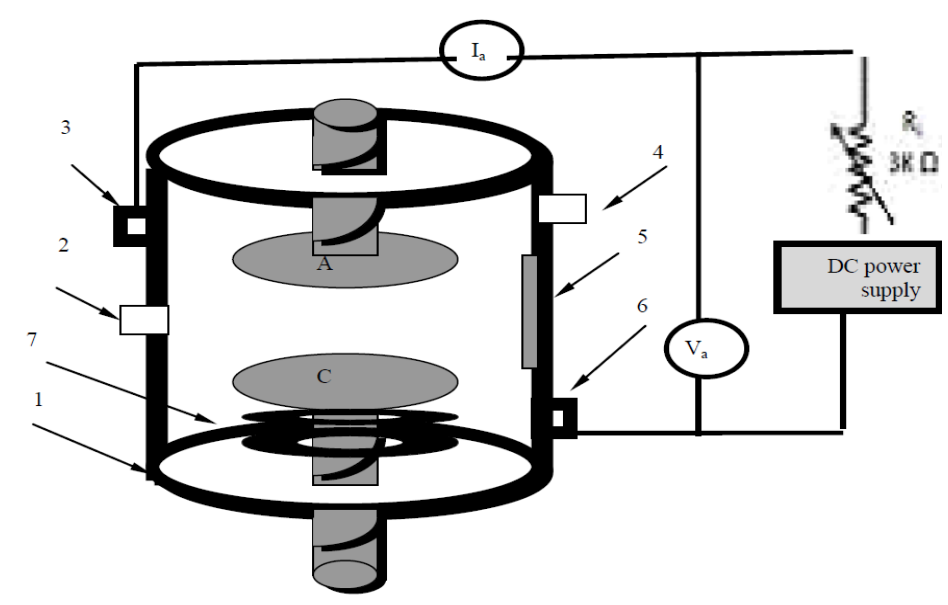

(a)

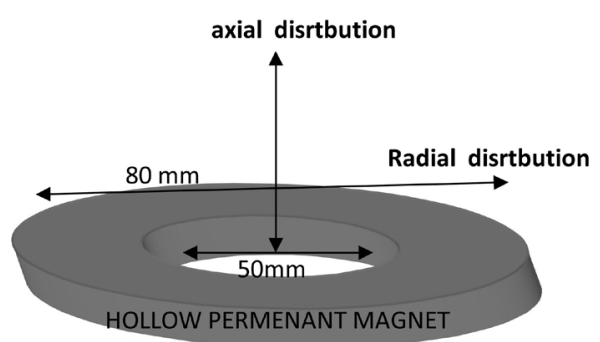

(b)

Figure 1. (a) The magnetized DC electrical discharge circuit. (C) movable cathode; (A) movable anode; (1) cylindrical metal envelope; (2) Helium gas in; (3) connected with anode metal plate; (4) gas out; (5) circular glass window for probes and spectrometer; (6) connected with cathode metal plate; (7) two permanent magnet; (b) Geometry and dimension of the hollow permanent magnets. 


\section{c. Methods}

Our method depends on suspend $111 \mathrm{~g}$ of the medium in one liter of purified water. Heat with frequent agitation and boil for one minute to completely dissolve the medium. Autoclave at $121^{\circ} \mathrm{C}$ for 15 minutes. Staphylococci will grow on this medium, while the growth of most other bacteria will be inhibited. Coagulase-positive staphylococci will produce luxuriant growth of yellow colonies and may have a yellow halo around the colony. Coagulase-negative staphylococci will produce small colorless to pink colonies with no color change to the medium.

\subsection{The Survival Curve}

Generally survival curves take different shapes [11] (single slope, Two-Slope, Three-Slopes) depending on the type of microorganism, the type of the gases supporting the microorganisms, and the relationship between $\mathrm{CFU} / \mathrm{ml}$ (cfu: colony-forming unit) and the exposure (reaction) time. Quantifying Sterilization Efficacy due to Equation (1):

$$
\text { Effect of Serilization }=\log _{10}\left[\frac{\text { Population before treatment }}{\text { Population after treatment }}\right]
$$

where $\mathrm{N}_{0}$ is the initial population, and $\mathrm{N}_{\mathrm{s}}$ is the surviving population.

\subsection{Experimental Conducts for Microbiological Samples with Plasma}

Viable suspension of Staphylococcus aureus was spread-plated onto slides containing nutrient agar under the influence of many parameters such as magnetic field strength, discharge power, gas pressure, and exposure time. The tests were conducted as follows:

1) Staphylococcus aureus is used in the present study. An overnight culture containing approximately $10^{8}$ cell-forming unit per milliliter (cfu/ml) was prepared. One of the slides was kept as control, whereas the others were exposed to the helium plasma discharge for different parameters. The plasma was in contact with the agar (the plasma discharge cover the slides). After exposure, the slides were incubated overnight at $37^{\circ} \mathrm{C}$ for $24 \mathrm{hrs}$.

2) At different exposure times, the series of the inoculated slides containing five slides were exposed to helium discharge, constant magnetic field strength of 400 Gauss, $3 \mathrm{~mJ}$ discharge power, and 0.53 mbar.

3) At different applied magnetic field strengths of 200 and 400 Gauss, constant exposure time of $30 \mathrm{~min}, 3 \mathrm{~mJ}$ discharge power, and 0.53 mbar.

4) At different applied power range (0.5, 1.5, and $3 \mathrm{~mJ})$, constant magnetic field strength of 400 Gauss, exposure time of $30 \mathrm{~min}$, and $0.53 \mathrm{mbar}$.

5) At different applied pressures of 0.27 and 0.53 mbar, constant magnetic field strength 400 Gauss, $3 \mathrm{~mJ}$ discharge power, exposure time of $30 \mathrm{~min}$, and $0.53 \mathrm{mbar}$.

6) Two plates were kept as control, by not exposing to the previous conditions.

\section{Results and Discussion}

\subsection{Distribution of the Magnetic Field Strengths}

Figure 2 shows the radial distribution of the magnetic field strength B measured for two different magnets with two different maximum magnetic field strengths of 200 and 400 Gauss, respectively, by using the Hall probe across the diameter of the cathode surface from one edge to the other. It observed that for the radial distribution, $\mathrm{B}$ was maximum at the two cathode edges and minimum at the center of the cathode and hollow magnets. Moreover for the axial distribution, B was maximum at the cathode surface, then it decreases far from the cathode, i.e. towards the anode (passing through the negative glow and positive column regions).

\subsection{Breakdown Potentials}

Figure 3 and Figure 4 show the comparison between $\left(\mathrm{I}_{\mathrm{a}}-\mathrm{V}_{\mathrm{a}}\right)$ characteristics of the helium gas discharge in the absence and under influence of magnetic field at pressures 0.27 mbar and 0.53 mbar respectively, as shown the starting potential decreased in the presence of the magnetic field. This is related to the increase of the mean free path, and the factor of decreasing the starting potential for helium, which may be attributed to the larger ionization 


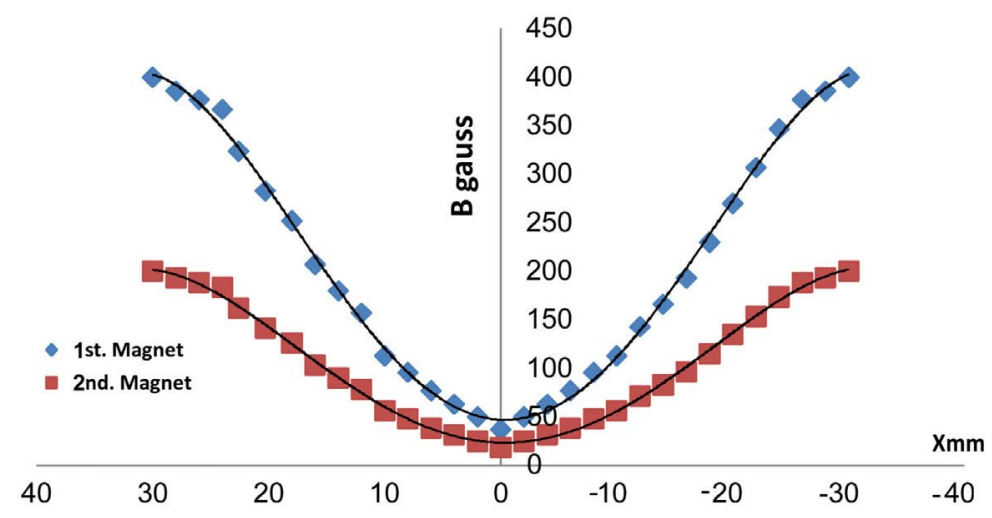

Figure 2. The radial distribution of magnetic field strength.

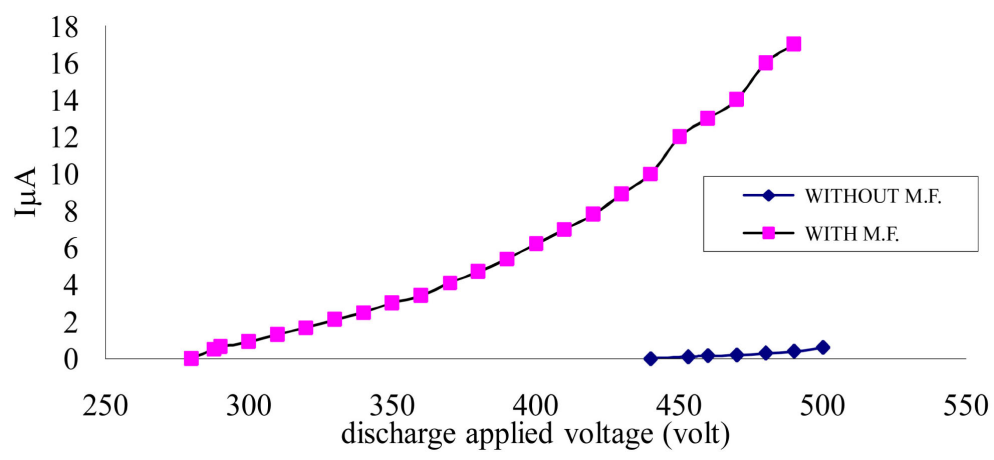

Figure 3. $\left(\mathrm{I}_{\mathrm{a}}-\mathrm{V}_{\mathrm{a}}\right)$ characteristics in the absence and under influence of magnetic field at pressure 0.27 mbar.

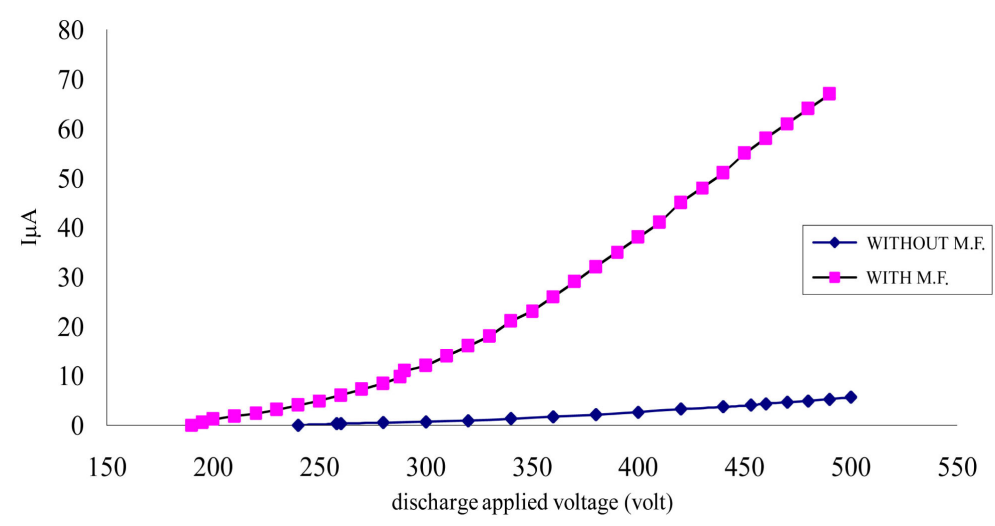

Figure 4. $\left(\mathrm{I}_{\mathrm{a}}-\mathrm{V}_{\mathrm{a}}\right)$ characteristics in the absence and under influence of magnetic field at pressure 0.53 mbar.

tion potential of helium. Moreover, values of the current discharge in the presence of the magnetic field for helium is larger than those in the absence of the field; thus the slopes of the curves are larger (i.e., the resistance of the discharge is small in the presence of the magnetic field) [12].

For helium, at small pressures in the absence of the magnetic field ( $\mathrm{P}=0.27$ and $0.53 \mathrm{mbar})$, it was difficult to initiate the gas breakdown, although it was initiated in the presence of the magnetic field; however, in the same conditions, breakdown does not occur for helium gas, whereas it may be occurs for some gases such as for argon gas. This can be attributed to the higher ionization potential of helium $(24.7 \mathrm{eV})$ than argon $(15.76 \mathrm{eV})$, that is, if helium pressure is increased, the breakdown voltage can be reduced over the same distance between the two electrodes [13]. 


\subsection{The Power Characteristic Curves of the Helium Gas Discharge}

As shown in Figure 5, in the absence of the magnetic field, the power characteristic curves of helium gas at pressures ranging from 0.27 to 4 mbar was between 0.3 and $25 \mathrm{~mJ}$. When the external magnetic field is applied as shown in Figure 6 for the same parameters, the curves are similar to those without magnetic field but the power range increased to between 0.38 and $48 \mathrm{~mJ}$. The reasons for the increase in the power under the influence of the magnetic field can be summarized in the following discussion:

1) When the magnetic field is applied, the cathode fall (CF) and negative glow regions are compressed, resulting in decreased length of the CF region $\left(\mathrm{d}_{\mathrm{c}}\right)$. Therefore, higher values of potential are expected. This will, of course, increase the electric field and the power in this region. Consequently, ions would accelerate more and efficient sputtering would increase, as well as the increase in the density of the plasma. Thus an increase in the magnetic field, the power, and the plasma density renders it compatible with many processes such as inactivation process for alive cells of microorganisms [14].

2) According to guidelines of the International Commission on Non-Ionizing Radiation Protection (ICNIRP), nonhealing and nonelective ultraviolet radiant exposure of the skin in the spectral region $180-400 \mathrm{~nm}$ should not exceed $3 \mathrm{~mJ} / \mathrm{cm}^{2}$. This value should be applied for the most sensitive, nonpathologic skin phototype (known as "melano-compromised") [15].

Then by reason of our results from our previous study for argon discharge [16] and this study on helium discharge, As shown in Figure 5, in the absence of the magnetic field, the power range was $0.3-2.8 \mathrm{~mJ}$ for applied pressure 0.53 - 1 mbar, suitable as per ICNIRP guidelines and Figure 6 shows that in the presence of the magnetic field, the power range was $1.5-3 \mathrm{~mJ}$ for applied pressure $0.27-0.53 \mathrm{mbar}$, which is also suitable for ICNIRP. Therefore, UV spectrum of helium discharge at low pressure as shown in Figure 7 is suitable for inactivation process as per the guidelines of ICNIRP, impact on skin at low pressures is well investigated particularly in dermatology because excess UV exposure causes detrimental effects on skin.

\subsection{The Radial Distribution of the Electron Temperature and Density}

The electron temperature and densities can be measured using the double probe method [17] [18], the double probe was moved radially from the edge to the center of the cathode (i.e., its direction perpendicular to the direction of the electric field lines), at different pressures ranges to investigate the radial distribution for CF region specially (in terms of the place of the treatment and disinfection of microbes). Figure 8 and Figure 9 show the temperature and density distribution of the CF region in the presence and in the absence of the magnetic field at different pressures (at center and edge) respectively. These figures clearly show the distribution profiles of $T_{e}$, where $T_{e}$ is decreased, $N_{e}$ continues to increase for the CF region. There is clearly a general trend that shows that values of $T_{e}$ and $N_{e}$ are inversely proportional $\left(T_{e} \times N_{e}=\right.$ constant); the maximum value of one is found where the minimum value of the other is seen.

Under the influence of the magnetic field on $\mathrm{T}_{\mathrm{e}}$ and $\mathrm{N}_{\mathrm{e}}$ for the CF region, the plasma is very intense (very thin bright ring) [19] [20], where the radial distributions of the electron density has its highest value at the edge, whereas the magnetic field is maximum, as shown in Figure 8. The electron temperature changes very little. The density begins to increases at the CF region at the edge more than at the center, as shown in Figure 9. This may be related to the distribution of the magnetic field, also because of the "edge effect" that caused because of the concentration of the electric field near the edge of the cathode [21].

\subsection{Cathode Fall Thickness}

It is noticed in our previous study [22] [23] that a reduction of the cathode fall (CF) thickness $\left(\mathrm{d}_{\mathrm{c}}\right)$ (about 37\%) for helium and $33 \%$ for argon occurs in the presence of the magnetic field at the edge of the cathode, as shown in Figure 10. Thus, a stronger electric field is produced and consequently more acceleration of the ions is expected that enhances the sputtering processes rate of the edge cathode surface. Moreover, values of $T_{e}$ decreased by increasing the gas pressure, whereas $\mathrm{N}_{\mathrm{e}}$ increased by increasing the gas pressure. This may be attributed to the increase in the number of electron-atom collisions and consequently, increasing the rate of ionization at higher pressure.

In the applied of the magnetic field, the diffusion coefficient and temperature of the electron reduced from the values given in the absence of the magnetic field. Furthermore, the electron density was observed to increase in 


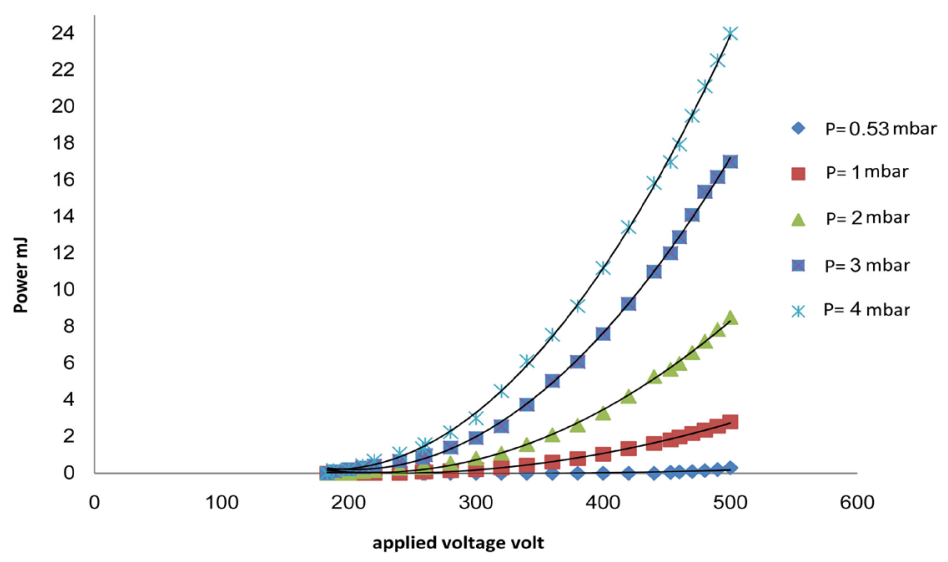

Figure 5. The power characteristic curves of the helium gas discharge in the absence of M.F.

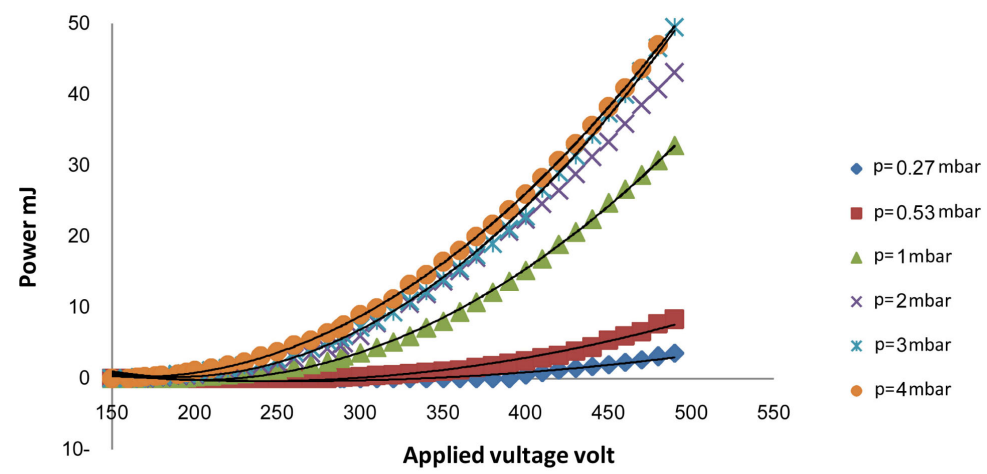

Figure 6. The Power characteristic curves of the Helium gas discharge in the Presence of M.F.

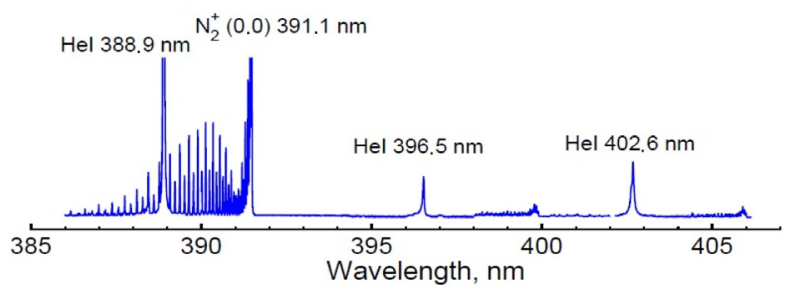

Figure 7. UV spectrum of helium discharge at low pressure in the presence of magnetic field.

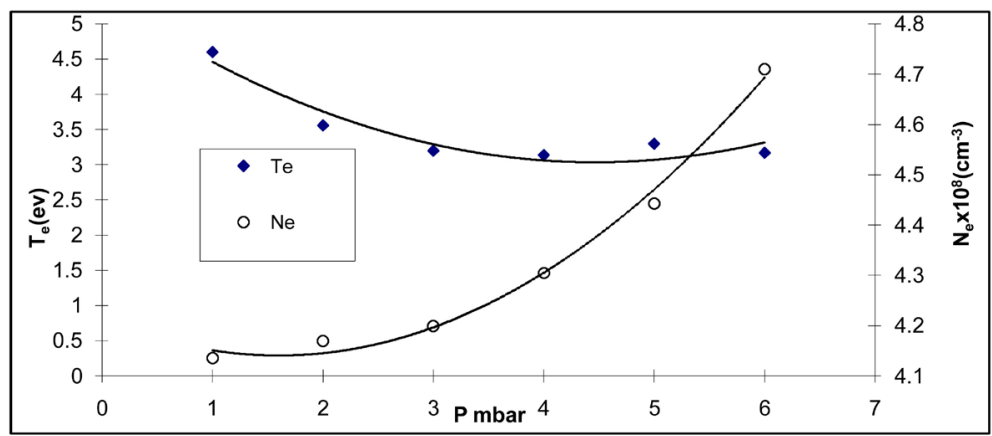

Figure 8. The temperature and density distribution of cathode fall region in the presence of M.F. at different pressures (at center). 


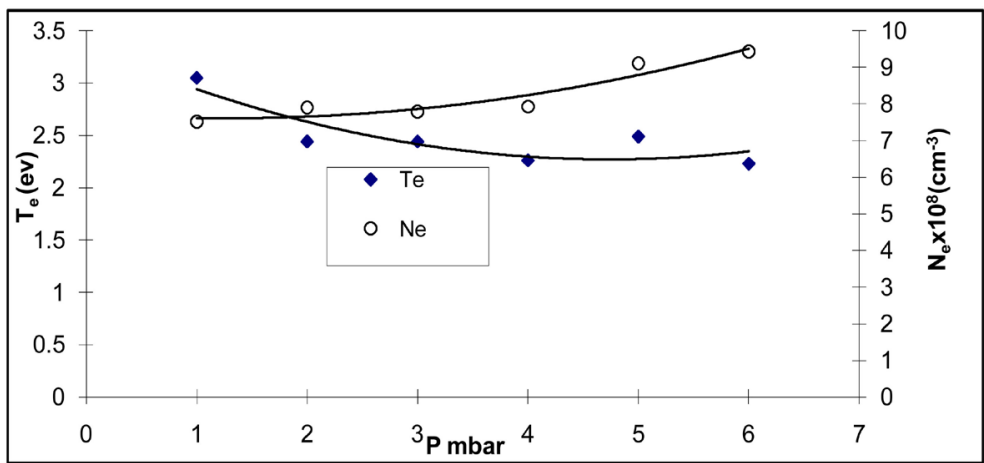

Figure 9. The temperature and density distribution of cathode fall region in the presence of M.F. at different pressures (at edge).

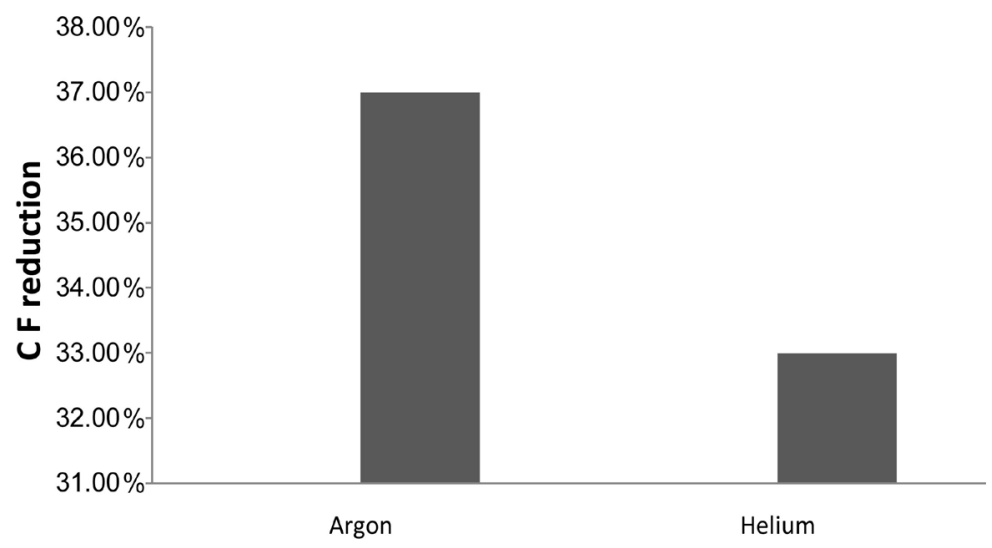

Figure 10. The effect of the magnetic field on the reduction of the cathode fall.

the presence of the magnetic field, where the radial distributions of the electron density were high at the edge with higher magnetic field strength than at the center as shown before through Sections 3 and 4.

\subsection{Survival Curves}

In this study, our target for helium discharge is the survival curves under magnetized DC plasma at the cathode edge. The results were focused on the influence of many parameters such as magnetic field strength, discharge power, gas pressure, and exposure time.

Figure 11 shows the inactivation process of Staphylococcus aureus bacteria. Moreover Figure 12 shows the effect of magnetic field strength on inactivation process after exposure time of $30 \mathrm{~min}$, where the residual survived microbes ratio reach $45 \%$ for applied 200 Gauss and decreased to $10 \%$ for 400 Gauss.

Furthermore, Figure 13 shows that the bacterial inactivation rate increased with increasing the power range from $(0.5 \mathrm{~mJ}$ to $3 \mathrm{~mJ})$, where the inactivation process increased as the discharge power range increased. Moreover, Figure 14 shows the survival curves of E-coli spores under influnce of 400 Gauss at different applied gas pressures of 0.27 and 0.53 mbar. For each case, there are two phases [24] [25]: phase one determines the fact that radicals from the gaseous phase are involved because of the given limited penetration depth of UV photons of plasma discharge. Because of the radicals, electrons, and positive ions, the destruction of isolated alive cells by UV photons dominates during this phase; phase two is partly dependent on the erosion rate of the various materials (coats, debris, dead cells) covering the still alive cells. Finally, the survival curves for helium in the gas pressure $0.53 \mathrm{mbar}$ has less number of alive cells $(\mathrm{CFU} / \mathrm{mL})$ than those under the influence of $0.27 \mathrm{mbar}$. This means that the presence of the magnetic field increased the ability of low pressure plasmas and their density to inactivate bacteria. In addition, there is an impressive inhibitory effect of plasma discharge for the residual survival microbes. 


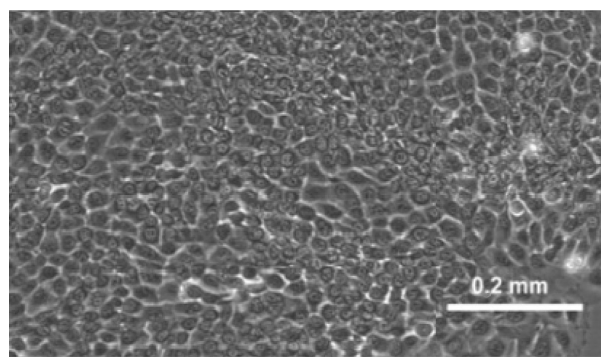

Figure 11. Inactivation process of Staphylococcus aureus bacteria.

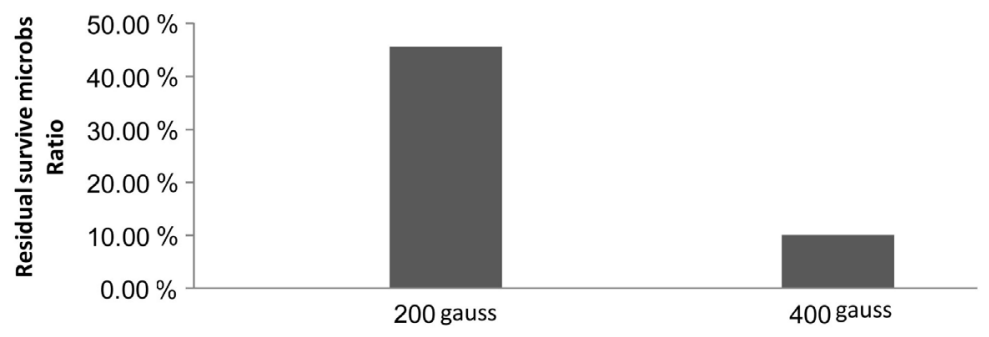

Figure 12. The effect of M.F. strength on inactivation process after exposure time $30 \mathrm{~min}$.

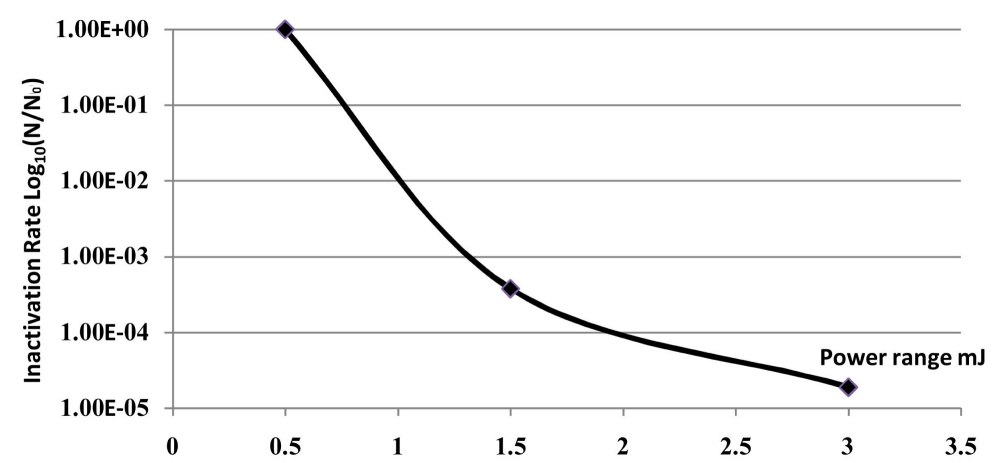

Figure 13. The bacterial inactivation rate with the power range (MJ).

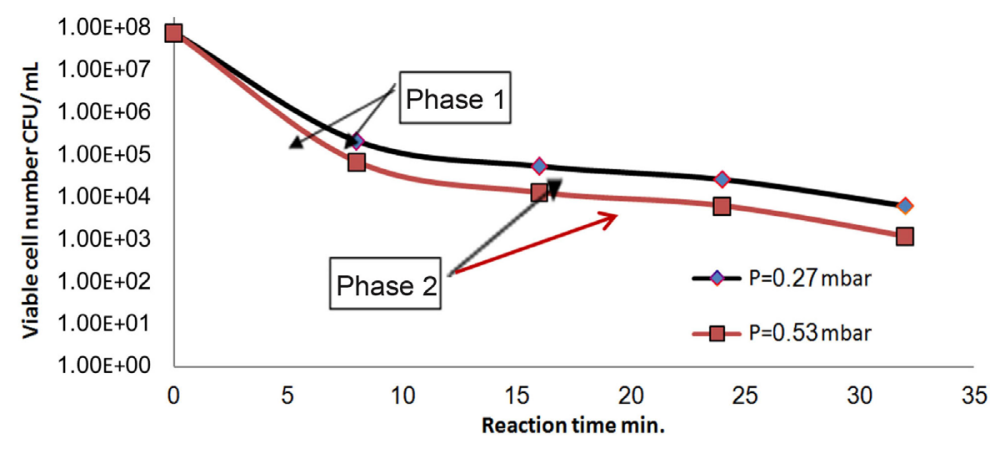

Figure 14. survival curves of Staphylococcus aureus spores under influnce of 400 gauss.

\section{Conclusions}

Magnetic field is a new trend and necessary to help the cold plasma to disinfection of bacteria for short exposure times and completely sterilize, to be able to eliminate viable cells at very short application times. Under the in- 
fluence of magnetized DC plasma, there are many evidences of its effect such as:

1) When magnetic field is applied, the CF and negative glow regions are compressed, therefore, higher values of potential are expected.

2) A magnetic field is applied such that field lines enter and leave through the cathode plate. Gas ions in the plasma are unconfined by the magnetic field. They are accelerated toward the cathode and strike it at high energy. In addition to sputtering Staphylococcus aureus target, the impact of ions produces secondary electron emission. These electrons are accelerated back into the plasma and are confined near the cathode by the magnetic field.

3) Because of the increase in magnetic field strength $B$, the Larmor radius $\left[L_{R}\right]$ decreases, and the diffusion coefficient across the field is reduced, and therefore, the electron temperature decreases. Moreover, the density of the plasma increases and becomes denser over the Staphylococcus aureus microbes in the same region of the effected magnetic field, especially at the edge more than the center. When using the magnetic field, the exposure time decreases to approximately half the value compared to those in the absence of the magnetic field.

4) Increasing magnetic field strength, low gas pressure, low discharge power, edge effect, and plasma density are very important parameters affecting the inactivation process of microbes and survival curves behavior. Anyway, sterilization is achieved in all cases; inactivation of bacterial cells is ultimately the result of the DNA destruction by UV photons. Finally, in the survival curves under the influence of the magnetic field, there are two phases that are sufficiently cleared from debris for the UV photons to finally kill them.

\section{References}

[1] Laroussi, M., Mendis, D.A. and Rosenberg, M. (2003) New Journal of Physics, 5, 41. http://dx.doi.org/10.1088/1367-2630/5/1/341

[2] Soloshenko, I.O., Khomich, V.A., Tsiolko, V.V., et al. (1999) Theoretical and experimental study of the factors of the sterilization of medical articles in low pressure glow discharge plasma Proceedings of the 14th International Symposium on Plasma Chemistry, Prague, 2-6 August 1999, 2551-2556.

[3] Soloshenko, I.A., Tsiolko, V.V. and Khomich, V.A. (2000) Plasma Physics Report, 26.

[4] Lu, X., et.al. (2008) Journal of Applied Physics, 104, Article ID: 053309. http://dx.doi.org/10.1063/1.2977674

[5] Singh, M.K., Ogino, A. and Nagatsu, M. (2009) New Journal of Physics, 11, Article ID: 115027. http://dx.doi.org/10.1088/1367-2630/11/11/115027

[6] Vicoveanu, D., Ohtsu, Y. and Fujita, H. (2008) Japanese Journal of Applied Physics, 47, 1130. http://dx.doi.org/10.1143/JJAP.47.1130

[7] Laroussi, M. (2002) IEEE Transactions on Plasma Science, 30, 1409-1415. http://dx.doi.org/10.1109/TPS.2002.804220

[8] Kakikawa, M. and Yamada, S. (2012) IEEE Transactions on Magnetics, 48, 2869-2872. http://dx.doi.org/10.1109/TMAG.2012.2200881

[9] United States Pharmacopeial Convention (2007) The United States Pharmacopeia. 31st Edition, The United States Pharmacopeial Convention, Rockville.

[10] Directorate for the Quality of Medicines of the Council of Europe (EDQM) (2007) The European Pharmacopoeia. Council of Europe, Strasbourg.

[11] Laroussi, M. and Leipold, F. (2004) International Journal of Mass Spectrometry, 233, 81-86. http://dx.doi.org/10.1016/j.ijms.2003.11.016

[12] Bohm, D. (1949) The Characteristics of Electrical Discharge in Magnetic Fields. McGraw-Hill, New York.

[13] Chapman, B. (1980) Glow Discharges Processes. Wiley, New York.

[14] Laroussi, M. (2005) Plasma Processes and Polymers, 2, 391-400.

[15] ICNIRP - International Commission on Non-Ionizing Radiation Protection (2004) Health Physics, 87, 171-186. http://dx.doi.org/10.1097/00004032-200408000-00006

[16] Galaly, A.R. and El Akshar, F.F. (2013) Physica Scripta, 88, Article ID: 065503.

[17] Chen, F.F. (1965) Plasma Diagnostic Techniques. Academic, New York.

[18] Brown, S.C. (1966) Introduction to Electrical Discharges in Gases. Wiley, New York.

[19] Spasojević, D., Steflekova, V., Šišović, N.M. and Konjević, N. (2012) Plasma Sources Science and Technology, 21, Article ID: 025006. http://dx.doi.org/10.1088/0963-0252/21/2/025006 
[20] Simonchikpp, L., Pitchfordpp, L. and Safronau, Y. (2012) Effect of the Cathode Surface Temperature on the Cathode Fall Layer Parameters ESCAMPIG xxi Conference, Viana do Castelo, 10-14 July 2012.

[21] Yasuda, H.K., Tao, W.H. and Prelas, M.A. (1996) Journal of Vacuum Science and Technology A, $14,2113$. http://dx.doi.org/10.1116/1.580089

[22] Galaly, A.R. and El Akshar, F.F. (2013) Physica Scripta, 88, Article ID: 065503.

[23] Galaly, A.R. (2014) Determination of the Cathode Fall Thickness in the Magnetized DC Plasma for Helium Gas Discharge. Proceedings of the IMEPS, International Middle East Plasma Science Conference, Belek-Antalya, 23-25 April 2014.

[24] Galaly, A.R. and Zahran, H.H. (2013) Journal of Physics: Conference Series (IOP), 431, Article ID: 012014.

[25] Galaly, A.R. (2014) The Edge Effect of Magnetized DC-Plasma for E-coli Inactivation. Proceedings of the IMEPS, International Middle East Plasma Science Conference, Belek-Antalya, 23-25 April 2014. 
Scientific Research Publishing (SCIRP) is one of the largest Open Access journal publishers. It is currently publishing more than 200 open access, online, peer-reviewed journals covering a wide range of academic disciplines. SCIRP serves the worldwide academic communities and contributes to the progress and application of science with its publication.

Other selected journals from SCIRP are listed as below. Submit your manuscript to us via either submit@scirp.org or Online Submission Portal.
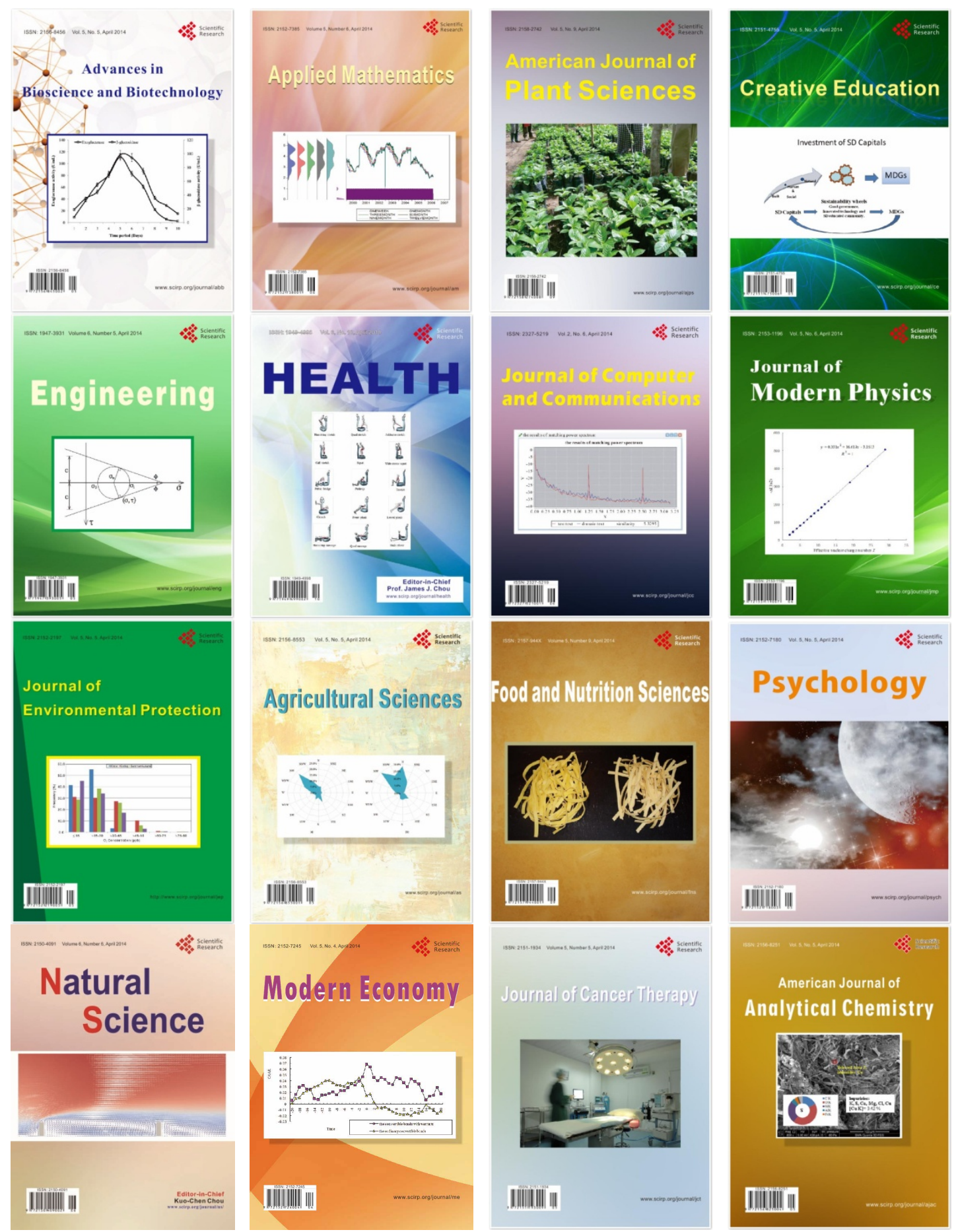